\title{
Improving glycemic and cholesterol control through an integrated approach incorporating colesevelam - a clinical perspective
}

\author{
Ronald B Goldberg \\ Division of Endocrinology, Diabetes \\ and Metabolism, Diabetes Research \\ Institute, University of Miami Miller \\ School of Medicine, Miami, FL, USA
}

Correspondence: RB Goldberg I450 NW IOth Ave, Miami,

FL 33136, USA

Tel +l 3052436505

Fax +I 305 243-526I

Email rgoldber@med.miami.edu

\begin{abstract}
Bile sequestrants have been used for almost 50 years to lower low density lipoprotein cholesterol (LDL-C). The advent of colesevelam in 2000 provided a more tolerable add-on LDL-C-lowering agent with an excellent safety record and with likely benefit for coronary heart disease events. Colesevelam lowers LDL-C approximately $15 \%$, and has an additive effect when combined with statin or non-statin lipid-modifying agents. It also tends to increase triglyceride levels. The discovery that bile sequestrants also lower glucose levels led to definitive large-scale clinical trials testing the effect of colesevelam as a dual antihyperglycemic agent with LDL-Clowering properties in type 2 diabetic subjects on metformin-, sulfonylurea- or insulin-based therapy with inadequate glycemic control. Colesevelam was found to lower hemoglobin A1c (HbA1c) by approximately $0.5 \%$ compared to placebo over the 16 - to 26 -week period, and had similar effects on the lipid profile in these diabetic subjects, as had previously been demonstrated in non-diabetic individuals. Colesevelam was well tolerated, with constipation being the most common adverse effect, and did not cause weight gain or excessive hypoglycemia. Colesevelam thus combines antihyperglycemic action with LDL-C-lowering properties, and should be useful in the management of type 2 diabetes.
\end{abstract}

Keywords: colesevelam, treatment, hyperglycemia, LDL-cholesterol

\section{Introduction}

Evidence indicates that effective treatment of hyperglycemia, and dyslipidemia ameliorates the occurrence and severity of the vascular complications of type 2 diabetes. ${ }^{1-3}$ At a time where diabetes prevalence is expanding significantly world-wide, it is critical that every effort be made to achieve recommended treatment targets for vascular risk factors as early on in the course of diabetes as possible. The American Diabetes Association (ADA) has recommended an $\mathrm{HbA} 1 \mathrm{c}$ target of $<7.0 \%$ as the target for good glycemic control, and in individual patients, a goal as close to normal (HbA1c $<6.0 \%$ ) without occurrence of hypoglycemia, ${ }^{4}$ while the American Association of Clinical Endocrinologists (AACE) has recommended an HbA1c goal of $<6.5 \%$. ${ }^{5}$ The primary lipid goal for people with diabetes recommended by the National Cholesterol Education Program is to achieve a LDL-C value of $<100 \mathrm{mg} / \mathrm{dL}$, and for very high-risk subjects of $\leq 70 \mathrm{mg} / \mathrm{dL} .{ }^{6}$ Based on the most recent surveys a large proportion of subjects with type 2 diabetes are not at their HbA1c and LDL-C goals. ${ }^{7,8}$

It has become abundantly clear that as glycemic and lipid treatment goals have become more stringent, a requirement for early combination therapy is needed in many patients. While this is self-evident in severely hyperglycemic patients, it is not always appreciated that the absolute glucose-lowering efficacy of a single oral antihyperglycemic agent is smaller in subjects who have milder degrees of hyperglycemia. ${ }^{9}$ Thus even in subjects with more modest degress of hyperglycemia, 


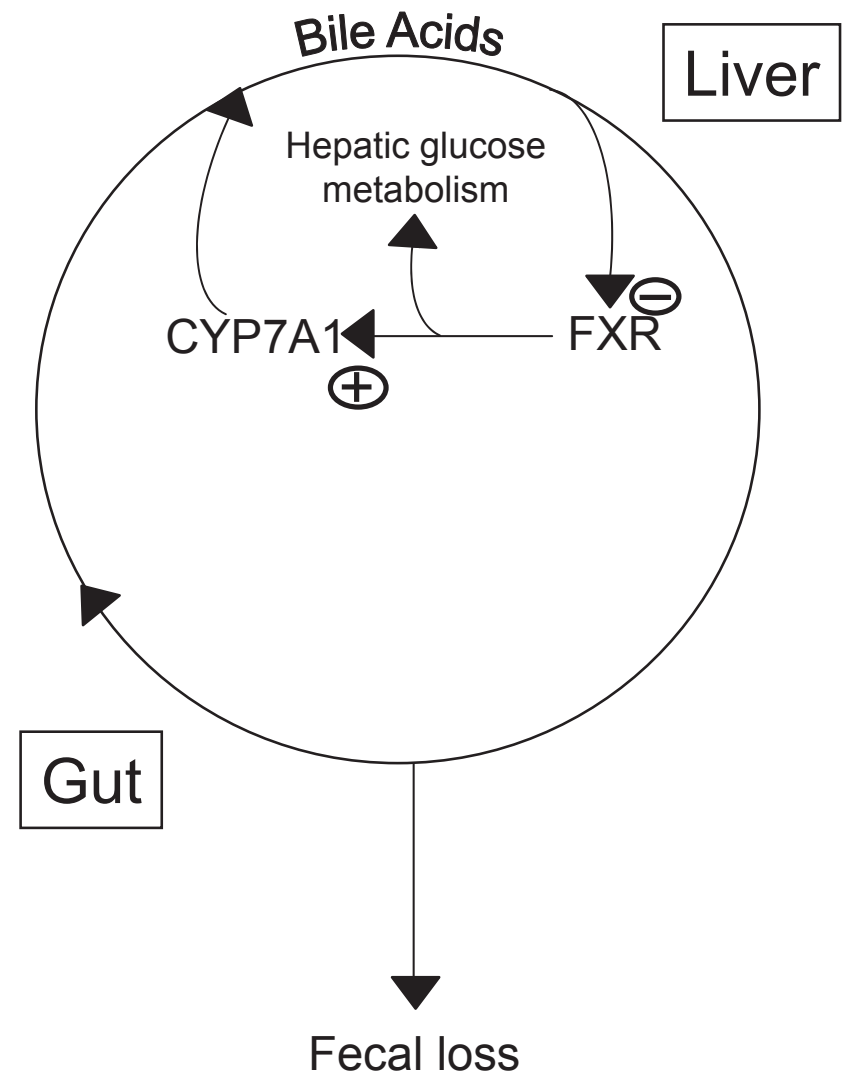

Figure I The enterohepatic circulation of bile acids.

Abbreviations: FXR, farnesoid $X$ receptor; CYP7AI, cholesterol $7 \alpha$-hydroxylase.

add-on treatment to monotherapy, usually metformin, is frequently required. This approach has been embodied in the recently published titration protocols developed by the ADA and the AACE for the treatment of hyperglycemia. ${ }^{10,11}$ Similarly it is being increasingly appreciated that an LDL-C target of $\leq 70 \mathrm{mg} / \mathrm{dL}$ often requires $\geq 50 \%$ reduction in LDL-C levels, an effect that may not always be achieved with maximum doses of statin montherapy. Thus add-on treatment with secondary LDL-C-lowering agents is frequently required as well. These needs have stimulated drug development and this has led to the introduction of several new antihyperglycemic and LDL-C-lowering agents which will require long-term evaluation for safety and health efficacy. It is in this context that the recent introduction of a second generation bile sequestrant, colesevelam, as an antihyperglycemic agent with LDL-lowering properties needs to be appreciated.

\section{Initial use of bile acid sequestrants as LDL-C-lowering agents}

Colesevelam was first introduced as an LDL-C-lowering drug in 2000. This followed the long-standing use of bile acid binding resins for treatment of hypercholesterolemia beginning almost 50 years ago. ${ }^{12}$ As their cholesterol-lowering properties were appreciated, the bile sequestrants became the initial choice for lowering of elevated LDL-C. The first resin available was colestyramine, to be followed a few years later by colestipol. These are positively charged, non-absorbable, anion-exchange, long-chain polymers that bind the anionic bile acids, and in so doing disrupt the enterohepatic circulation of bile acids, depleting the bile acid pool by about $40 \%$. Normally approximately $5 \%$ of the bile acid pool is lost daily in the stool, an amount that is replaced by new bile acid synthesis from hepatic cholesterol, initiated by the action of the rate limiting enzyme of bile acid synthesis, cholesterol $7 \alpha$ hydroxylase. This daily synthesis of bile acids accounts for a sizable portion of daily cholesterol turnover (about $50 \%$ ) and is regulated through the action of the farnesoid $X$ receptor (FXR) in the liver on cholesterol $7 \alpha$ hydroxylase. Bile acids are the primary ligand of FXR and inhibit its activity, leading to suppression of the hydroxylase. Thus bile acid sequestration will result in loss of FXR-mediated suppression of cholesterol $7 \alpha$-hydroxylase, leading to a rise in its activity which diverts hepatic cholesterol to bile acid synthesis. The fall in hepatocyte cholesterol concentration then results in an upregulation of LDL receptors and a fall in circulating LDL-C levels. Cholestyramine in doses of 4 to $24 \mathrm{~g}^{13}$ and colestipol 2 to $16 \mathrm{~g}$ daily ${ }^{14}$ were shown to lower LDL-C in a dose-dependent manner by $5 \%$ to $28 \%$, and in the case of colestipol, apo B was reduced in parallel by $2 \%$ to $28 \% .{ }^{14}$ Both agents caused a modest increase in triglyceride levels.

A major barrier to the more widespread use of these two first generation sequestrants is their unpalatable gritty consistency when the powder is ingested as a slurry with liquid. In addition constipation and flatulence are common particularly with higher doses. The introduction of statins in the late 1980s, which were both more potent as well as more tolerable than bile acid sequestrants, quickly displaced these agents as first choice treatment for lowering LDL-C. Despite this, the limitations of statin therapy in patients with severe hypercholesterolemia in whom a minimum of $50 \%$ LDL-C lowering was needed, were soon appreciated. Several studies were published in the early 1990s demonstrating the value of add-on cholestyramine or colestipol to treatment with, pravastatin, ${ }^{15}$ fluvastatin ${ }^{16}$ and lovastatin, ${ }^{17}$ in which approximately $50 \%$ lowering of LDL-C and up to $40 \%$ lowering of apo $\mathrm{B}^{18}$ was achieved. Furthermore combination of these agents with non-statin drugs such as niacin ${ }^{19}$ or fibrates ${ }^{20}$ provided added LDL-C-lowering capability to triglyceride-lowering or HDL-C-raising agents, without the safety concerns that had been raised by combined use of 


\section{Cholestyramine}

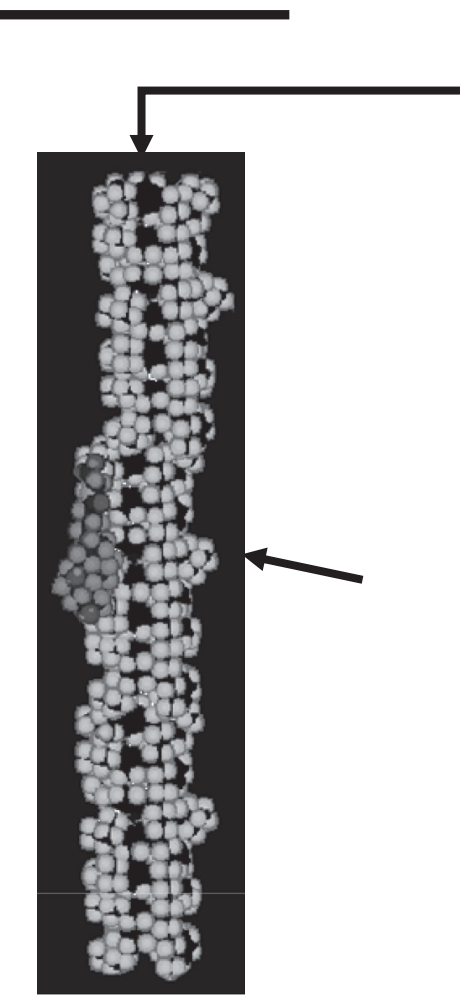

Colesevelam HCI

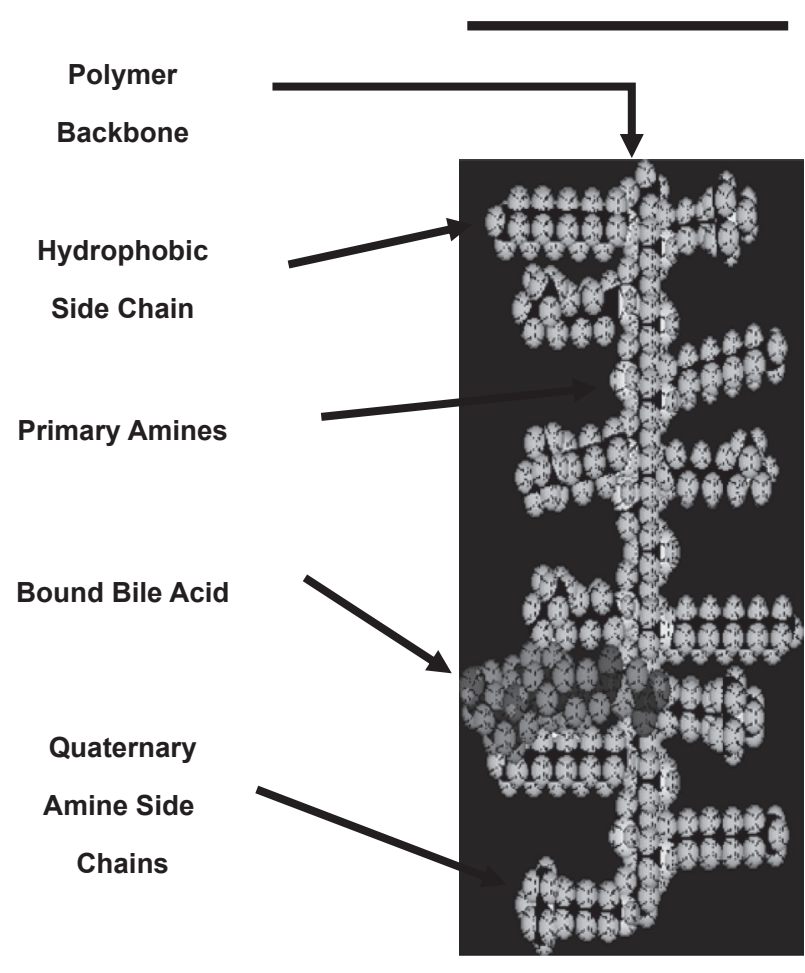

Figure 2 Comparison of the structures of cholestyramine and colesevelam.

these drugs with statins. The large body of published clinical trials data, the record of safety, the characterization of their adverse effects, and the decades-long experience with first generation bile acid sequestrant therapy in the treatment of hypercholesterolemia ${ }^{21}$ are important factors in evaluating the place of colesevelam in the management of type 2 diabetes because of the similarities in pharmacology and mechanisms of action among all bile acid sequestrants.

\section{Evidence that bile acid sequestrants prevent cardiovascular disease}

In recent years concerns about the effects of antihyperglycemic agents on cardiovascular disease (CVD) are coming in to focus. The issue was initially raised by the University Group Diabetes Program which found that the biguanide phenformin and the sulfonylurea tolbutamide were associated with increased occurrence of cardiovascular disease. ${ }^{22}$ This led to the withdrawal of phenformin, and the requirement for a black box warning for all sulfonylurea and subsequently meglitinide drugs. There has been little subsequent evidence that sulfonylurea drugs increase CVD, and the remaining biguanide, metformin, in a single small study was associated with a reduced occurrence of myocardial infarction. ${ }^{23}$
However a recent meta-analysis of clinical trials using rosiglitazone has once again raised concern that some antihyperglycemic agents may have deleterious effects on CVD. ${ }^{24}$ Because CVD is of such importance in the prognosis of type 2 diabetes, this issue has received great attention, such that the US Food and Drug Agency has recently recommended that long-term clinical trial assessments of effects of all new antihyperglycemia agents on CVD incidence will now be mandatory. ${ }^{25}$ This requirement is also probably necessary for all lipid-lowering agents used in long-term diabetes management, as has been recently highlighted by the finding that fenofibrate did not significantly reduce the primary CVD outcome in a large population with type 2 diabetes. ${ }^{26}$

In this setting it is worth briefly reviewing the available evidence that bile acid depletion therapy has beneficial effects on CVD occurrence. The first indication that lowering of LDL-C through biliary diversion could successfully reduce CVD events comes from the Program on the Surgical Control of the Hyperlipidemias (POSCH) ${ }^{27}$ In this study of 838 men and woman with hypercholesterolemia who had survived a first myocardial infarction, biliary diversion and LDL-C reduction was achieved by means of partial ileal bypass surgery. LDL-C levels were reduced by $37.7 \%$ 


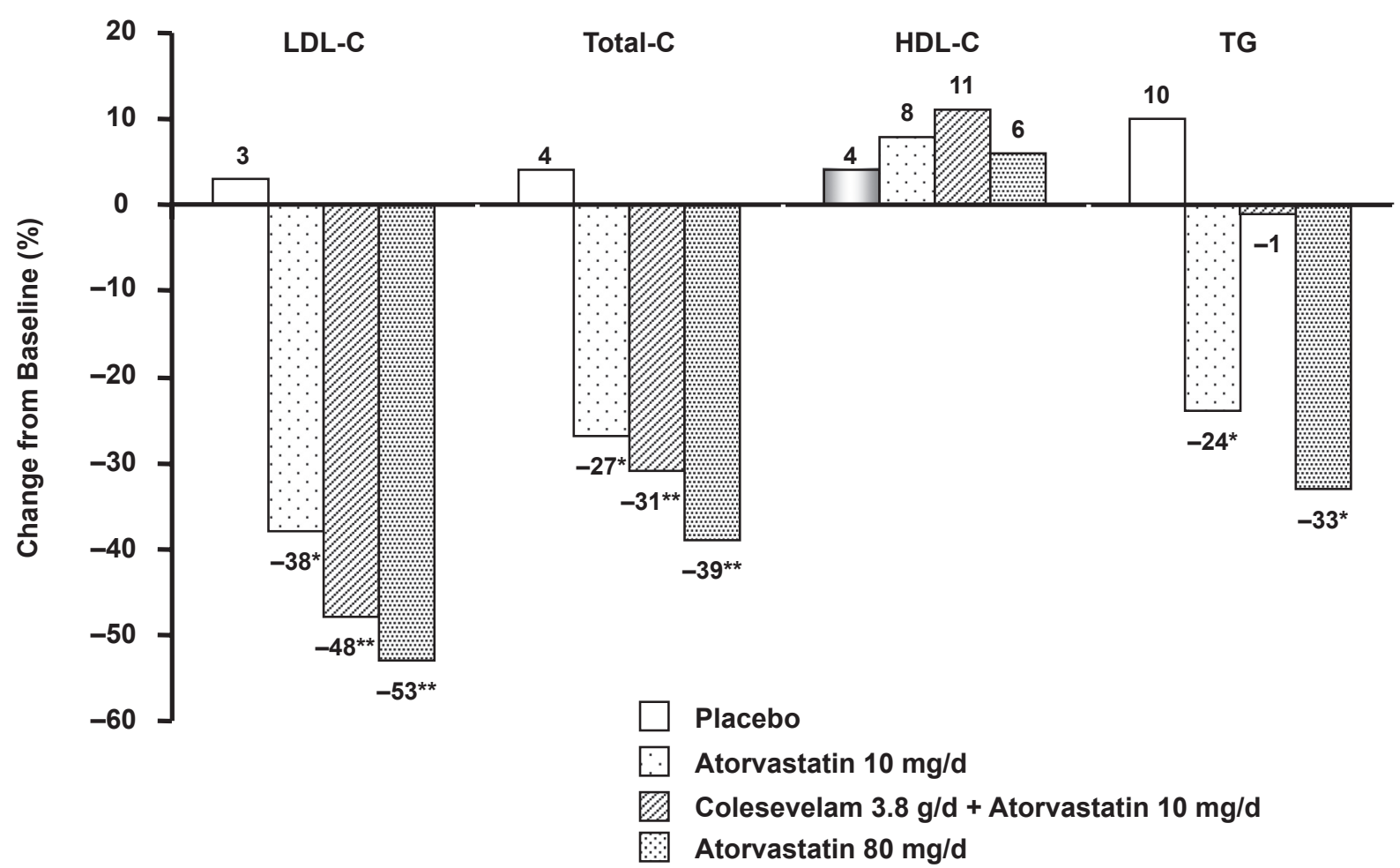

Figure 3 Percentage change in total cholesterol, low-density lipoprotein (LDL) cholesterol, high-density lipoprotein (HDL) cholesterol, and triglyceride (TG) levels from baseline (week 0 ) to the end of treatment (week 6) in patients who received placebo or colesevelam hydrochloride therapy. Reproduced with permission from Davidson MH, Dillon MA, Gordon B, et al Colesevelam hydrochloride (Cholestagel): a new, potent bile acid sequestrant associated with a low incidence of gastrointestinal. side effects. Arch Intern Med. 1999;159:1893-1900. ${ }^{32}$ Copyright @ 1999 American Medical Association. All rights reserved.

over a 5 -year period compared to that in the control group and this was accompanied by a significant $35 \%$ reduction in combined coronary heart disease (CHD) mortality and non-fatal myocardial infarction. In the modern context, this study is highly relevant because it constitutes clinical trial evidence supporting the contention that lowering of LDL-C can explain most if not all of the beneficial effect on CVD in the long-term clinical trials with statins. The strongest evidence that the effects of surgically induced biliary diversion are mirrored by sequestrant-induced biliary depletion comes from the Lipid Research Clinics Coronary Primary Prevention Trial (LRC-CPPT) ${ }^{13}$ and the NHLBI Type II Coronary Intervention Study. ${ }^{28}$ In the CPPT, one of the first large studies to definitively associate a drug-induced reduction in LDL-C with a reduction in CHD risk, 3806 men were randomized to cholestyramine or placebo for a mean of 7.4 years; the cholestyramine group had a $19 \%$ reduction in CHD risk $(\mathrm{p}<0.05)$ that was associated with a $13 \%$ decrease in LDL-C levels. ${ }^{13}$ The NHLBI Type II Coronary Intervention Study ${ }^{28}$ evaluated the effects of cholestyramine versus placebo on the progression of coronary angiographic lesions after 5 years of treatment in 116 patients. LDL-C levels were reduced by $26 \%$ in the cholestyramine and $5 \%$ in the placebo groups $(\mathrm{p}<0.001)$ and coronary lesions progressed in $49 \%$ of placebo-treated patients versus $32 \%$ of cholestyramine-treated patients $(p<0.05)$. There have also been several secondary intervention trials using bile acid sequestrants in combination with niacin, statins and/or fibrates which were uniformly and in some cases dramatically positive, but in which it was not possible to tease out the effect of the bile acid sequestrants from the other agents used. ${ }^{19,29,30}$ Finally a meta-analysis of 8 bile acid sequestrant studies found that monotherapy significantly reduced cardiac mortality. ${ }^{31}$ While there have been no such intervention trials with colesevelam, the evidence to date supports the notion that lowering of LDL-C through bile acid depletion is associated with beneficial effects on CHD.

\section{Colesevelam as an LDL-C- lowering agent}

Although colesevelam acts in the same way as do cholestyramine and colestipol it exhibits several important differences from them. First, colesevelam was developed to have enhanced specificity, greater affinity, and higher capacity for binding bile acids than cholestyramine or colestipol. This was achieved by engineering long hydrophobic side chains 


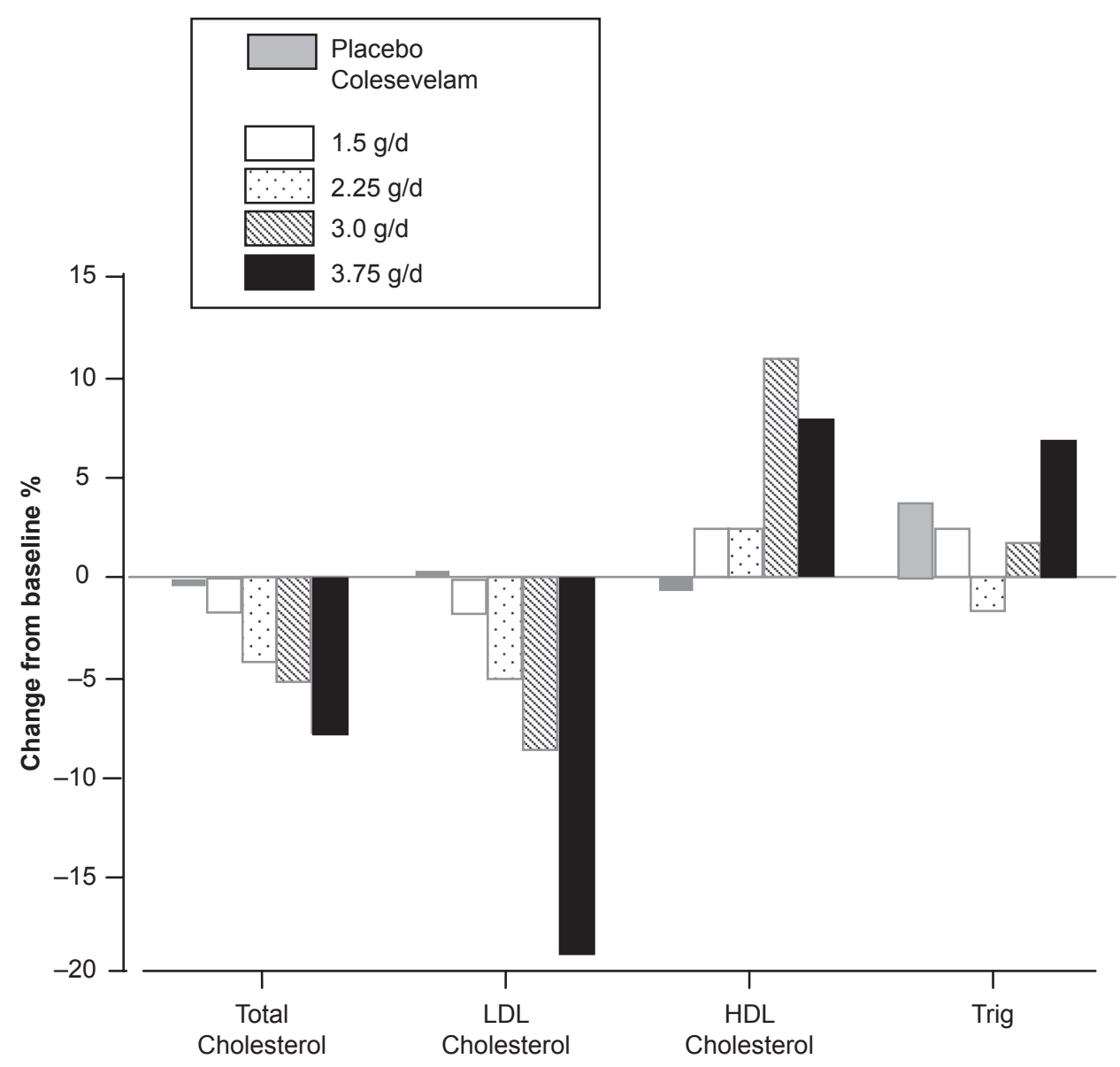

Figure 4 Effect of addition of colesevelam to atorvastatin $10 \mathrm{mg}$. LDL-C and Total- $C$ values are expressed as mean; HDL-C and TG values are expressed as median; ${ }^{*}$ P $<0.05$ vs placebo **p $<0.05$ vs atorvastatin $10 \mathrm{mg}$. Reproduced with permission from Hunninghake D. Insull W Jr., Toth P. et al, Coadministration of colesevelam hydrochloride with

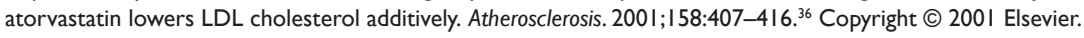

to the backbone polymer, which maximize hydrophobic interactions with bile acids, adding to the effects of the ionic bonds that bind bile acids in the two first generation compounds. Thus colesevelam is at least 2 to 3 times more potent on a weight basis than either cholestyramine or colestipol. Hence smaller amounts of colesevelam can be used effectively, with less adverse effects, and with greater tolerability through its packaging into tablets instead of a slurry of liquefied powder. Although the usual dose requires taking 6 to 7 tablets $(625 \mathrm{mg} /$ tablet $)$ per day, this is still considerably more acceptable than the ingestion of an insoluble powder several times a day. Finally there is no need for titration of the dose of colesevelam to allow for tolerability, unlike that commonly required by first generation sequestrants.

\section{Monotherapy}

The first of the clinical trials with colesevelam evaluating its efficacy as an LDL-C-lowering agent, utilized a double-blind, placebo-controlled, dose ranging design (1.5, $2.25,3.0$, or $3.75 \mathrm{~g} /$ day), conducted in 137 patients for 6 weeks. Colesevelam lowered LDL-C levels significantly and dose-dependently by up to $19 \%$, increased HDL-C by up to $8 \%$ to $9 \%$ and no significant changes were observed in triglyceride levels. ${ }^{32}$ Similar findings were obtained in a 24-week study which demonstrated that LDL-C reduction occurred within 2 weeks, was sustained over 6 months and was paralleled by up to a $12 \%$ reduction in apo B. ${ }^{33}$

\section{Combination therapy with statins}

Colesevelam has been studied in combination studies with small doses of lovastatin $(10 \mathrm{mg})$, simvastatin (10 and $20 \mathrm{mg}$ ), and atorvastatin $(10 \mathrm{mg})$, in which LDL-C levels were lowered respectively by up to $34 \%, 42 \%$ and $48 \%{ }^{34-36}$ Although it is usually recommended that the statin dose should be up-titrated before a second agent is added if greater LDL-C lowering is needed, actual or potential adverse statin effects may limit the dose that can be used, in which case 


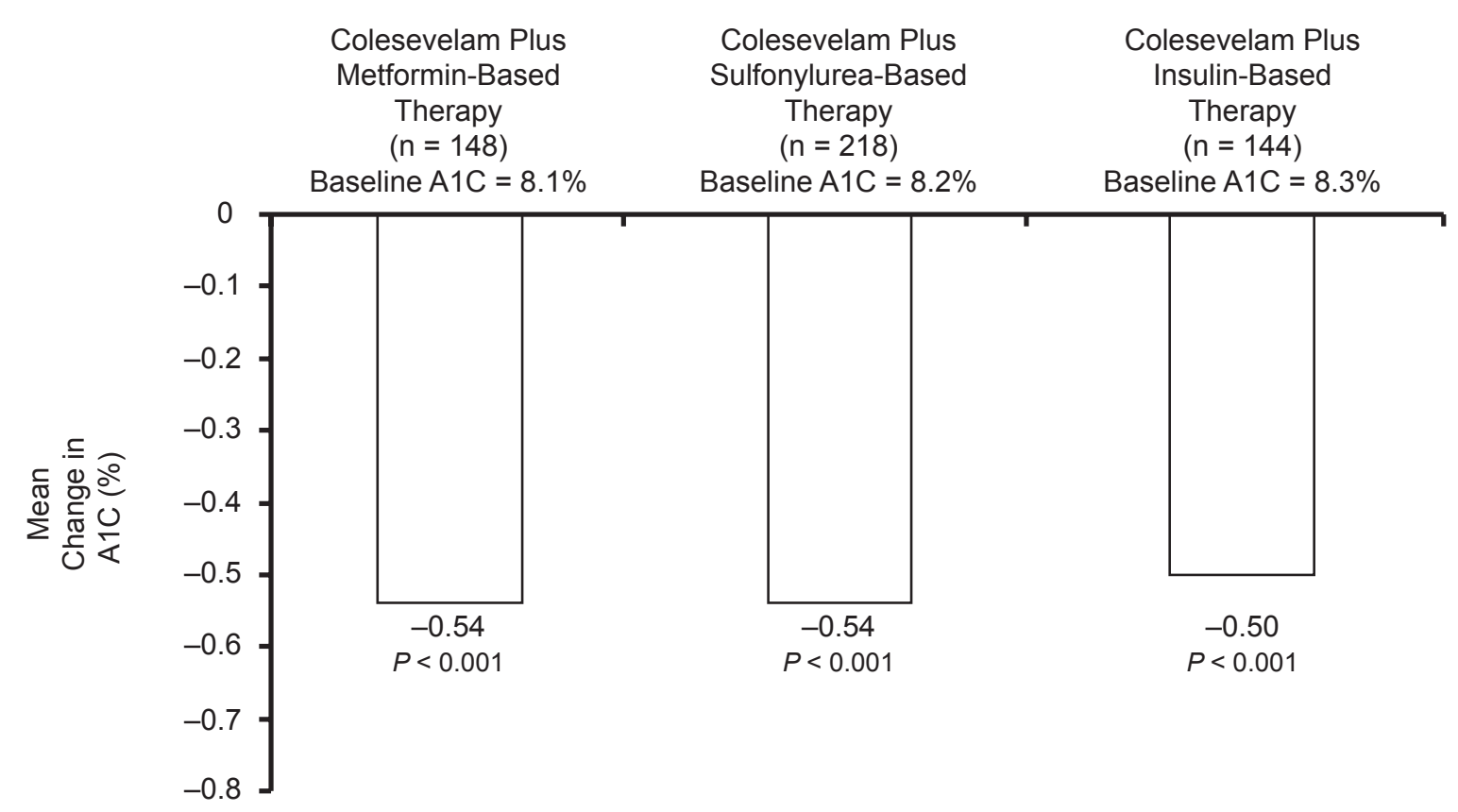

Figure 5 Summary of effect of add-on colesevelam $3.75 \mathrm{~g} /$ day to metformin-, sulfonylurea-, and insulin-based treatment on the placebo-controlled HbAlc reduction at study end from baseline, in subjects with type 2 diabetes (intention to treat population, last observation carried forward). . $1,50,51^{4}$

the addition of colesevelam significantly adds to the effect of low dose statins.

\section{Effects of colesevelam on other lipoproteins and lipoprotein subfractions}

Bile sequestrants in general and colesevelam in particular appear to increase HDL-C and apo A-I levels modestly, perhaps through the deactivation of FXR, since FXR has been demonstrated to suppress apo A-I synthesis. ${ }^{37}$ Cholestyramine and colestipol have been shown to raise triglyceride levels, and this constitutes a concern in patients with type 2 diabetes in whom hypertriglyceridemia is common. Fortunately the effect of bile acid sequestrants on triglyceride levels is usually mild, though it can be dramatic in severely hypertriglyceridemic subjects ${ }^{38}$ and this is a contraindication to the use of bile sequestrants. The increase in triglyceride levels was shown to result from increased VLDL production, ${ }^{39}$ an effect that has been partially linked to the reduction of FXR activity by bile acid depletion. Bile acids, acting through FXR and hepatocyte nuclear factor 4, have been shown to reduce microsomal triglyceride transfer protein which initiates VLDL assembly. ${ }^{40}$ Although there have been no formal reports of colesevelam exacerbating severe hypertriglyceridemia, this is almost certainly a class effect and the use of colesevelam in severely hypertriglyceridemic subjects is contraindicated. Mild to moderate elevation of triglyceride levels with colesevelam have been described. ${ }^{41}$ Despite this tendency to increase triglyceride levels, colesevelam has been demonstrated to increase LDL size ${ }^{42}$ while decreasing LDL particle number predominantly by reducing the number of small LDL particles in a manner unrelated to triglyceride levels. ${ }^{43}$ This may be due to suppression of hepatic lipase activity as a result of the bile-acid sequestrant-induced upregulation of FXR, since FXR has been demonstrated to suppress hepatic lipase. ${ }^{44}$ Further studies are needed to understand this interesting phenomenon.

\section{Combination therapy with non-statin drugs}

Combination of colesevelam with ezetimibe may be helpful in subjects intolerant to statin treatment or in subjects who can tolerate only minimal amounts of statin. Colesevelam and ezetimibe have different modes of action and therefore might be expected to have additive effects on LDL-C. This was confirmed in several short-term studies in which the combined treatment lowered LDL-C by $30 \%$ to $40 \% .{ }^{45,46} \mathrm{In}$ combination with fenofibrate, ${ }^{47}$ colesevelam was shown to lower LDL-C (17\%), beyond that achieved with a fibrate, while the presence of the fibrate assured that there would be net triglyceride reduction (32\%). This approach with or 

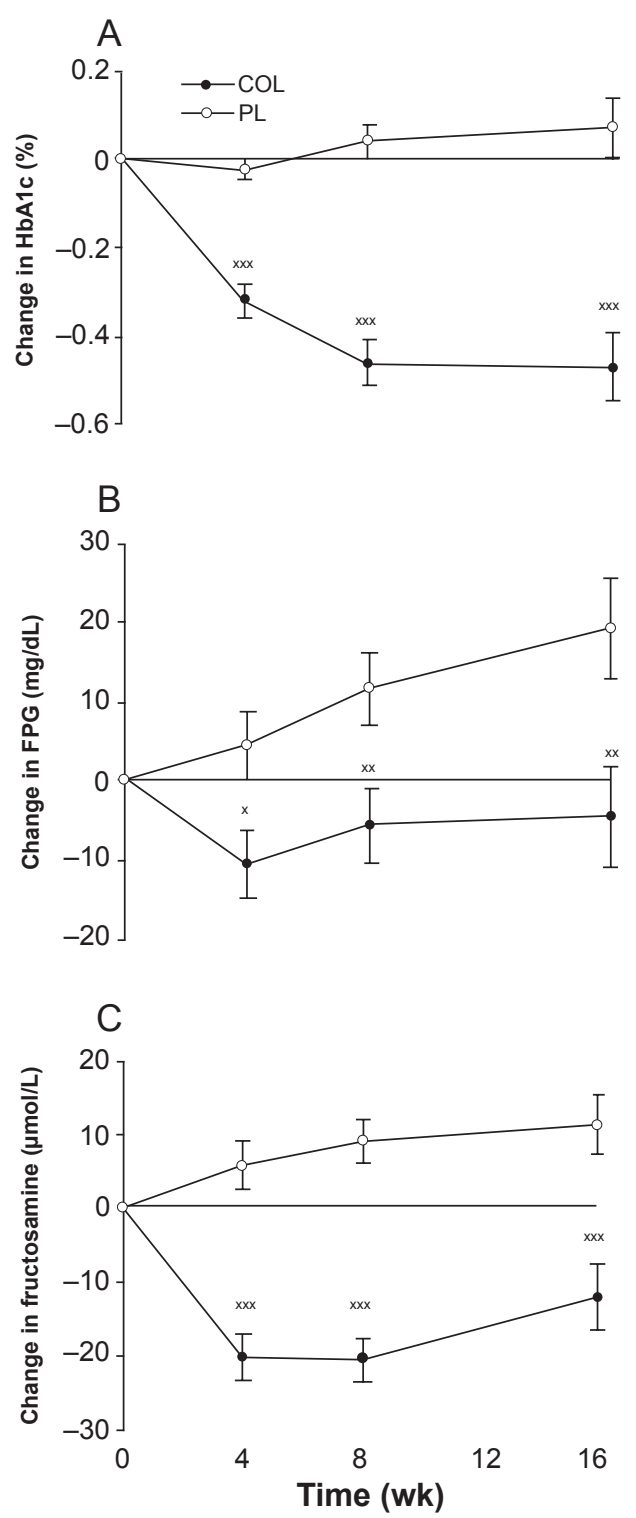

Figure 6 Least squares mean (SEM) change from baseline in glycated hemoglobin A Ic ( $\mathrm{HbAlc}$ ) A), fasting plasma glucose (FPG) B), and fructosamine C) levels in subjects (intent-to-treat population without last observation carried forward imputation) receiving colesevelam hydrochloride, $3.75 \mathrm{~g} / \mathrm{d}$, or placebo for 16 weeks. Goldberg RB Fonseca VA, Truitt KE, Jones MR. Efficacy and safety of colesevelam in patients with type 2 diabetes mellitus and inadequate glycemic control receiving insulin-based therapy. Arch Intern Med. 2008; 168(I4):153I-1540.41 Copyright (C) 2008 American Medical Association. All rights reserved.

Abbreviations: FPG, fasting plasma glucose; COL, colesevelam; PL, placebo.

without added ezetimibe may be useful if there was a need to avoid or minimize statin therapy in subjects with mixed dyslipidemia.

\section{Colesevelam, a new antihyperglycemic agent}

The effect of bile sequestrants to lower glucose levels was first noted in 1994 in a study of cholestyramine in treatment of dyslipidemia in type 2 diabetes. ${ }^{48}$ Several subsequent studies produced conflicting results, but the Glucose Lowering Effect of Welchol study (GLOWS) clearly demonstrated that colesevelam improved glycemia in 65 type 2 diabetic subjects inadequately controlled on oral antihyperglycemic agents and randomized to $3.75 \mathrm{~g}$ of colesevelam versus placebo for 12 weeks. ${ }^{49}$ Colesevelam treatment was associated with an $\mathrm{HbA} 1 \mathrm{c}$ reduction of $0.5 \%$ from a baseline value of $7.9 \%$ to $8.1 \%$ compared to placebo. In addition there was a reduction in those receiving colesevelam in fasting glucose at 4 and 8 weeks and postprandial glucose at 12 weeks, as well as fructosamine levels, from baseline compared to placebo. In subjects with a baseline $\mathrm{HbAlc} \geq 8.0 \%$, the difference between colesevelam and placebo treatment was reduced by $1.0 \%$. These findings in this pilot study led to the larger pivotal trials for the indication of the use of colesevelam for treatment of hyperglycemia in type 2 diabetes.

\section{The pivotal trials testing a glucose- lowering indication for colesevelam}

Three similarly designed, concurrent, prospective, multicenter, randomized, placebo-controlled parallel-group lasting 16 to 26 weeks were conducted between August 2004 and July 2006, testing the effects of colesevelam on glycemic and lipid measures in type 2 diabetic subjects inadequately controlled on sulfonylurea, ${ }^{50}$ metformin $^{51}$ and insulin ${ }^{41}$ treatments respectively. Since these are the only largescale clinical trials with the primary objective of assessing the antiglycemic properties of colesevelam in established type 2 diabetic subjects that have been published, they are discussed below in detail. The subjects enrolled had an $\mathrm{HbA} 1 \mathrm{c}$ between $7.5 \%$ to $9.5 \%$, and in the metformin and sulfonylurea studies were respectively on stable doses of either metformin or a sulfonylurea alone, or in combination with other oral antihyperglycemic agents; dipeptidyl protease IV inhibitor therapy was not included. In the insulin studies participants had to be taking insulin alone or in combination with oral agents. In all of the studies, individuals with an LDL-C $<60 \mathrm{mg}$, a triglyceride level $>500 \mathrm{mg} / \mathrm{dL}$ or with dysphagia, or swallowing or intestinal dysmotility disorders were among those excluded. Subjects were withdrawn from the study if their $\mathrm{HbA} 1 \mathrm{c}$ or fasting glucose at any visit exceeded $10 \%$ or $260 \mathrm{mg} / \mathrm{dL}$ respectively or they developed hypoglycemia. After a 2-week, single-blind run-in period, subjects were randomized 1:1 to colesevelam taken either as six $625 \mathrm{mg}$ tablets once daily or twice daily (total $3.75 \mathrm{~g}$ ) or matching placebo (according to the preferences of the study participant), added to their prestudy antihyperglycemic 
treatment. In the metformin and sulfonylurea-based treatment studies, assessments were made at 6, 12, 16 and 26 weeks, whereas in the insulin-based studies, these were done at 4 , 8 and 16 weeks; all were analyzed with the last observation carried forward. The primary efficacy parameter was the HbA1c change.

\section{Effect of colesevelam in patients with inadequately controlled type 2 diabetes on sulfonylurea-based therapy ${ }^{50}$}

Of 461 randomized subjects, 166 and 141 completed the study in the colesevelam and placebo group respectively. Their mean age was 57 years, $53 \%$ to $56 \%$ were men, a quarter were Hispanic and $10 \%$ to $15 \%$ African American, mean BMI was 33 and mean HbA1c was $8.2 \%$ to $8.3 \%$. About one third were on sulfonylurea monotherapy, and the remainder were receiving combination therapy with metformin or in about a quarter of these, with a thiazolidinedione. At week 26 colesevelam treatment resulted in a significant least squares mean $\mathrm{HbA} 1 \mathrm{c}$ reduction of $0.54 \%$ ( $<<0.001)$ from baseline compared to the placebo group. Similar results were observed in the sulfonylurea monotherapy $(-0.79 \%$, $\mathrm{p}<0.001)$ and sulfonylurea combination therapy subgroups $(-0.42 \%, \mathrm{p}<0.001)$. In addition both fasting glucose and fructosamine levels were reduced, with fasting glucose significantly below baseline at 6 weeks. There were more subjects in the colesevelam group with a glycemic control response - defined as achievement of either a $\mathrm{HbA} 1 \mathrm{c}$ reduction of $\geq 0.7 \%$ or a $\geq 30 \mathrm{mg} / \mathrm{dL}$ fasting glucose reduction (47\%) compared to the placebo group (32\%). Eighteen subjects in the colesevelam group and 40 in the placebo group met discontinuation criteria. The LDL-C fell by a net $16.7 \%$ and the triglyceride level rose by a net $17.7 \%$ in the colesevelam treated group; both were significant changes. The most common drug-related adverse event was constipation (6.1\% versus $2.6 \%$ in the placebo group) and 18 subjects in the colesevelam, versus 9 in the placebo group withdrew due to a drug-related adverse event. There was no excess of hypoglycemic events or weight gain in the treatment group.

\section{Effect of colesevelam in patients with inadequately controlled type 2 diabetes on metformin-based therapy ${ }^{51}$}

In this study 316 subjects were randomized, of whom 222 completed the study. Their baseline characteristics were very similar to those in the sulfonylurea-treatment based trials described above. About half were receiving metformin monotherapy, and of those on combination therapy over $85 \%$ were taking sulfonylurea drugs, and just under a half were treated with thiazolidinediones. Compared to placebo, colesevelam reduced the HbA1c by $0.54 \%(\mathrm{p}<0.001)$ at 26 weeks from a mean baseline HbA1c of $8.3 \%$; the net reduction was $0.47 \%$ for the monotherapy subgroup and $0.62 \%$ in those receiving combination therapy, and for those with a baseline $\mathrm{HbAlc}>8.0 \%$ the net reduction was $0.6 \%$. A significant $20.8 \mathrm{mg} / \mathrm{dL}$ reduction in the fasting glucose value was noted at 6 weeks at which time fructosamine levels had also been significantly lowered; both remained significantly lowered compared to placebo at 26 weeks. As in the sulfonylurea studies, a greater proportion of the colesevelam group achieved either a reduction in the $\mathrm{HbA} 1 \mathrm{c}$ of $\leq 0.07 \%$ or fasting glucose $\leq 30 \mathrm{mg} / \mathrm{dL}$ than placebo (48\% versus $35 \%$ ), and only 3 subjects in the colesevelam group met discontinuation criteria for hyperglycemia (or hypoglycemia) versus 16 in the placebo group. LDL-C levels fell by a significant net $15.9 \%$ from a baseline value of $99-106 \mathrm{mg} / \mathrm{dL}$, apo B by $8 \%(\mathrm{p}<0.001)$ and triglyceride concentrations increased from a baseline value of 166 to $172 \mathrm{mg} / \mathrm{dL}$ by a net $4.7 \%$ in the colesevelam group at week 26 (39\%-48\% were on statin treatment). The adverse event experience paralleled that in the sulfonylurea study.

\section{Effect of colesevelam in patients with inadequately controlled type 2 diabetes on insulin-based therapy ${ }^{41}$}

Of 287 insulin-treated patients randomized, 231 completed the study. Demographic characteristics of participants were very similar to those in the above two studies, although they were slightly more obese (mean BMI 35) and their mean total daily insulin dose was 74 to 78 units. Insulin therapy consisted of either basal insulin alone, insulin mixtures or true basal/bolus therapy, and about $40 \%$ were on insulin monotherapy. Over half of those on insulin/oral agent combination treatment received metformin, about a third of these subjects were treated with sulfonylurea, and just under a third were on a thiazolidinedione. The net treatment difference in $\mathrm{HbAlc}$ level from baseline to week 16 in the colesevelam group was $-0.50 \%$ versus the placebo ( $p<0.001)$; the effects were similar in those on insulin monotherapy $(-0.59 \%)$ versus those treated with insulin/oral agent combinations $(-0.44 \%)$ and were greater in those with a baseline $\mathrm{HbA} 1 \mathrm{c}$ $\geq 8.0 \%$ versus $<8.0 \%(-0.57 \%$ compared to $-0.38 \%)$. As in the previous studies, fasting glucose and fructosamine fell rapidly and were significantly reduced compared to 
placebo within 4 weeks, and there were more subjects with a glycemic control response in the colesevelam group (49\%) versus placebo $(32 \%)$. In this study discontinuations for hyperglycemia were similar in the two groups. The LDL-C was reduced by a net $12.8 \%(\mathrm{p}<0.001)$, apo B by a net $5.3 \%(\mathrm{p}<0.04)$ and triglyceride values increased by $21.5 \%$ (baseline triglyceride $155-167 \mathrm{mg} / \mathrm{dL}, \mathrm{p}<0.001$ ). In the colesevelam-treated group, the most frequently reported drug-related adverse events were constipation (6.8\%), dyspepsia (3.4\%), hypoglycemia (3.4\%), flatulence (2.0\%), and nausea (1.4\%), whereas in the placebo group, the most frequently reported adverse event was hypoglycemia (5.7\%). Five subjects in the colesevelam group withdrew because of a drug related adverse effect versus 2 in the placebo group and there was no weight gain in either group.

\section{Effects on insulin sensitivity and inflammatory biomarkers}

No significant changes were noted in fasting insulin level or HOMA-IR in the metformin study ${ }^{51}$ nor on fasting C-peptide levels in any of the studies as a result of colesevelam treatment. However high sensitivity CRP levels fell by $14.0 \%$ from baseline at 24 weeks in the colesevelam groups in both the sulfonylurea and metformin studies, and by $19 \%$ compared to placebo in a study of patients with mild hypercholesterolemia. ${ }^{52}$ There was no effect on adiponectin levels with colesevelam treatment. ${ }^{51}$

\section{Interactions with other drugs}

Although bile sequestrants are not absorbed and therefore will not influence hepatic metabolism of other drugs, they are positively charged resins and tend to bind to acidic drugs, interfering with their absorption. This effect is greatest with cholestyramine, less with colestipol and least with colesevelam. For example, no significant effects of colesevelam administration were found on the pharmocokinetics of six drugs with narrow therapeutic indices, whose absorption is interfered with by cholestyramine, namely valproic acid, digoxin, quinidine, verapamil, metoprolol and warfarin..$^{53}$ Furthermore no effects of coadministration of colesevelam on drug levels of lovastatin, fenofibrate, metformin, glipizide, repaglinide and pioglitazone have been found.$^{54}$ Colesevelam does interfere with absorption of glyburide, levothyroxine, oral contraceptives, and post-marketing reports suggest interactions with the therapeutic efficacy of phenytoin and warfarin. It is recommended that coadministration of colesevelam with drugs with narrow therapeutic indices, and fat-soluble vitamins be avoided $;{ }^{54}$ instead the administration of the sequestrant should be delayed by at least 4 hours after these medications are ingested.

\section{Perspectives on the utility of colesevelam as an antihyperglycemic agent in type 2 diabetes}

The three pivotal studies demonstrate that add-on colesevelam lowers the $\mathrm{HbA1}$ c by about $0.5 \%$ in subjects with an average $\mathrm{HbA} 1 \mathrm{c}$ of approximately $8.2 \%$ to $8.3 \%$, irrespective of whether the underlying treatment is metformin-, sulfonylurea-, or insulin-based. These findings suggest that the antihyperglycemic effect of colesevelam is independent of the nature of the medication to which it is added. This would further imply that the mechanism of the antihyperglycemic effect of colesevelam is additive to those of metformin or sulfonylureas and does not appear to enhance insulin action based on surrogate markers, although more definitive studies are needed to examine this question. The mechanism by which bile sequestrants in general and colesevelam in particular improve hyperglycemia is unknown. Sequestrants may interfere with intestinal glucose absorption, ${ }^{55}$ potentially enhance insulin release, ${ }^{56}$ and experimentally through the reduction of FXR activity, alter hepatic glucose metabolism, although studies have yielded conflicting results. ${ }^{57}$ Based on effects of colesevelam treatment on fasting glucose and fructosamine levels, its antihyperglycemic effect is complete within 4 weeks.

Colesevelam offers several advantages in subjects on oral antihyperglycemic therapy or insulin treatment whose $\mathrm{HbA} 1 \mathrm{c}$ and/or LDL-C are mildly or moderately elevated above their targets. First this treatment simultaneously lowered $\mathrm{HbAlc}$ and LDL-C toward therapeutic targets without causing weight gain or excessive hypoglycemia in the clinical trials. Second, this class of non-absorbable resins offers a good safety record. Thirdly both surgical and pharmacologic biliary diversion to lower LDL-C has been shown to reduce coronary atherosclerosis and coronary heart disease events. Dividing the dose into 3 tablets at the midday meal and 3 at the evening meal may offer the most convenient way to administer this relatively bulky medication. This regimen also avoids interactions with most other medications which are usually taken in the morning, since their absorption should be complete by the midday meal. Finally in an era where prevention of diabetes has been accepted as an important approach to ameliorating the epidemic of diabetes including the early initiation of pharmacotherapy in high risk prediabetic individuals, ${ }^{58}$ colesevelam would seem to offer several advantages, for the reasons discussed above. 


\section{Disclosures}

The author has received research grants and speaker honoraria from Daiichi Sankyo, Inc, Parsippany, New Jersey, USA.

\section{References}

1. Gaede P, Lund-Andersen H, Parving HH, Pedersen O. Effect of a multifactorial intervention on mortality in type 2 diabetes. $N$ Engl J Med. 2008;358(6):580-591.

2. Colhoun HM, Betteridge DJ, Durrington PN, et al. CARDS investigators. Primary prevention of cardiovascular disease with atorvastatin in type 2 diabetes in the Collaborative Atorvastatin Diabetes Study (CARDS): multicentre randomised placebo-controlled trial. Lancet. 2004;364(9435):685-696.

3. Nathan DM, Cleary PA, Backlund JY, et al. Diabetes Control and Complications Trial/Epidemiology of Diabetes Interventions and Complications (DCCT/EDIC) Study Research Group. Intensive diabetes treatment and cardiovascular disease in patients with type 1 diabetes. N Engl J Med. 2005;353(25):2643-2653.

4. American Diabetes Association. Standards of medical care in diabetes2008. Diabetes Care. 2008;31 Suppl 1:S12-S54.

5. AACE medical guidelines. Endocr Pract. 2007;12 Suppl 1:4-68.

6. National Cholesterol Education Program Expert Panel on Detection, Evaluation, and Treatment of High Blood Cholesterol in Adults: Executive Summary of the Third Report of the National Cholesterol Education Program (NCEP) Expert Panel on Detection, Evaluation, and Treatment of High Blood Cholesterol in Adults (Adult Treatment Panel III). JAMA. 2001, 285:2486-2497.

7. Hoerger TJ, Segel JE, Gregg EW, Saaddine JB. Is glycemic control improving in US. adults? Diabetes Care. 2008;31(1):81-86.

8. Jacobs MJ, Kleisli T, Pio JR, et al. Prevalence and control of dyslipidemia among persons with diabetes in the United States. Diabetes Res Clin Pract. 2005;70(3):263-269.

9. Bloomgarden ZT, Dodis R, Viscoli CM, Holmboe ES, Inzucchi SE. Lower baseline glycemia reduces apparent oral agent glucose-lowering efficacy: a meta-regression analysis. Diabetes Care. 2006;29(9):2137-2139.

10. Nathan DM, Buse JB, Davidson MB, et al. Medical management of hyperglycaemia in type 2 diabetes mellitus: a consensus algorithm for the initiation and adjustment of therapy: A consensus statement from the American Diabetes Association and the European Association for the Study of Diabetes. Diabetologia. 2009;52(1):17-30.

11. Jellinger PS, Davidson JA, Road maps to achieve glycemic control in type 2 diabetes mellitus: ACE/AACE Diabetes Road Map Task Force. Endocr Pract. 2007;13(3):260-268.

12. Van Itallie TB, Hashim SA, Crampton RS, Tennent DM. The treatment of pruritus and hypercholesteremia of primary biliary cirrhosis with cholestyramine. N Engl J Med. 1961;265:469-474.

13. The Lipid Research Clinics Coronary Primary Prevention Trial results. 1. Reduction in incidence of coronary heart disease. JAMA. 1984;251:351-364.

14. Hunninghake DB, Stein EA, Bremner WF, et al. Dose response study of colestipol tablets in patients with moderate hypercholesterolemia. Am J Ther. 1995;2:180-189.

15. Pan HY, DeVautt AR, Swiles BJ, et al. Pharmacokinetics and pharmacodynamics of pravastatin alone and with cholestyramine in hypercholesterolemia. Clin Pharmacol Ther. 1990;48:201-207.

16. Sprecher DL, Abrams J, Allen JW, et al. Low-dose combined therapy with fluvastatin and cholestyramine in hyperlipidemic patients. Ann Intern Med. 1994;120:537-543.

17. Denke MA. Grundy SM. Efficacy of low-dose cholesterol-lowering drug therapy in men with moderate hypercholesterolemia. Arch Intern Med. 1995;155:393-399.

18. Schrott HG, Stein EA, Dujovne CA, et al. Enhanced low-density lipoprotein cholesterol reduction and cost-effectiveness by low-dose colestipol plus lovastatin combination therapy. Am J Cardiol. 1995;75:34-39.
19. Blankenhorn DH. Nessim SA, Johnson RL, et al. Beneficial effects of combined colestipol-niaein therapy on coronary atherosclerosis and coronary venous bypass grafts. JAMA. 1987:257:3233-3240.

20. Houlston R, Quiney J, Watts GF, el al. Gemfibrozil in the treatment of resistant familial hypercholesterolaemia and type 111 hyperlipoproteinaemia. J R Soc Med. 1988;81:274-276.

21. Insull W Jr. Clinical utility of bile acid sequestrants in the treatment of dyslipidemia: a scientific review. South Med J. 2006;99(3):257-273.

22. Prout TE. A prospective view of the treatment of adult-onset diabetes with special reference to the University Group Diabetes Program and oral hypoglycemic agents. Med Clin North Am. 1971;55(4): 1065-1075.

23. Effect of intensive blood-glucose control with metformin on complications in overweight patients with type 2 diabetes (UKPDS 34). UK Prospective Diabetes Study (UKPDS) Group. Lancet. 1998;352(9131):854-865

24. Nissen SE, Wolski K. Effect of rosiglitazone on the risk of myocardial infarction and death from cardiovascular causes. $N$ Engl J Med. 2007;356(24):2457-2471.

25. Food and Drug Administration. Available at: http://www.fda.gov/bbs/ topics/NEWS/2008/NEW01928.html.

26. Keech A, Simes RJ, Barter P, et al. Effects of long-term fenofibrate therapy on cardiovascular events in 9795 people with type 2 diabetes mellitus (the FIELD study): randomised controlled trial. Lancet. 2005;366(9500):1849-1861.

27. Buchwald H, Varco RL, Matts JP, et al. Effect of partial ileal bypass surgery on mortality and morbidity from coronary heart disease in patients with hypercholesterolemia. Report of the Program on the Surgical Control of the Hyperlipidemias (POSCH). N Engl J Med. 1990;323(14):946-955.

28. Brensike JF, Levy RI. Kelsey SF, et al. Effects of therapy with cholestyramine on progression of coronary arteriosclerosis: results of the NHLBI Type II Coronary Intervention Study. Circulation. 1984;69:313-324.

29. Kane JP, Malloy MJ, Ports TA, et al. Regression of coronary atherosclerosis during treatment of familial hypercholesterolemia with combined drug regimens. JAMA. 1990;264:3007-3012.

30. Whitney EJ. Krasuski RA. Personius BE, et al. A randomized trial of a strategy for increasing high-density lipoprotein cholesterol levels: effects on progression of coronary heart disease and clinical events. Ann Intern Med. 2005;142:95-104.

31. Studer M, Briel M, Leimenstoll B, et al. Effect of different antilipidemic agents and diets on mortality: a systematic review. Arch Intern Med. 2005;165:725-730.

32. Davidson MH, Dillon MA, Gordon B, et al. Colesevelam hydrochloride (Cholestagel): a new, potent bile acid sequestrant associated with a low incidence of gastrointestinal side effects. Arch Intern Med. 1999; 159:1893-1900.

33. Insull W Jr, Toth P, Mullican W, et al. Effectiveness of colesevelam hydrochloride in decreasing LDL cholesterol in patients with primary hypercholesterolemia: a 24-week randomized controlled trial. Mayo Clin Proc. 2001;76:971-982.

34. Davidson $\mathrm{MH}$, Toth $\mathrm{P}$, Weiss $\mathrm{S}$, et al. Low-dose combination therapy with colesevelam hydrochloride and lovastatin effectively decreases low-density lipoprotein cholesterol in patients with primary hypercholesterolemia. Clin Cardiol. 2001;24:467-474.

35. Knapp HH, Schrott H, Ma P, et al. Efficacy and safety of combination simvastatin and colesevelam in patients with primary hypercholesterolemia. Am J Med. 2001;110:352-360.

36. Hunninghake D, Insull W Jr, Toth $\mathrm{P}$, et al. Coadministration of colesevelam hydrochloride with atorvastatin lowers LDL cholesterol additively. Atherosclerosis. 2001;158:407-416.

37. Claudel T, Sturm E, Duez H, et al. Bile acid-activated nuclear receptor FXR suppresses apolipoprotein A-I transcription via a negative FXR response element. J Clin Invest. 2002;109(7):961-971.

38. Crouse JR 3rd. Hypertriglyceridemia: a contraindication to the use of bile acid binding resins. Am J Med. 1987;83(2):243-248. 
39. Beil U, Crouse JR, Einarsson K, Grundy SM: Effects of interruption of the enterohepatic circulation of bile acids on the transport of very low density lipoprotein triglycerides. Metabolism. 1982;31:438-444.

40. Hirokane H, Nakahara M, Tachibana S, Shimizu M, Sato R. Bile acid reduces the secretion of very low density lipoprotein by repressing microsomal triglyceride transfer protein gene expression mediated by hepatocyte nuclear factor-4. J Biol Chem. 2004;279(44):45685-45692.

41. Goldberg RB, Fonseca VA, Truitt KE, Jones MR. Efficacy and safety of colesevelam in patients with type 2 diabetes mellitus and inadequate glycemic control receiving insulin-based therapy. Arch Intern Med. 2008;168(14):1531-1540.

42. Rosenson RS. Colesevelam HCl reduces LDL particle number and increases LDL size in hypercholesterolemia. Atherosclerosis. 2006;185(2):327-330.

43. Rosenson RS, Abby SL, Jones MR. Colesevelam $\mathrm{HCl}$ effects on atherogenic lipoprotein subclasses in subjects with type 2 diabetes. Atherosclerosis. 2008; [Epub ahead of print] PMID: 18996525.

44. Sirvent A, Verhoeven AJ, Jansen H, et al. Farnesoid X receptor represses hepatic lipase gene expression. J Lipid Res. 2004;45(11):2110-2115.

45. Xydakis AM. Guyton JR. Chiou P, et al. Effectiveness and tolerability of ezetimibe add-on therapy to a bile acid resin-based regimen for hypercholesterolemia. Am J Cardiol. 2004;94:795-797.

46. Zema MJ. Colesevelam $\mathrm{HCl}$ and ezetimibe combination therapy provides effective lipid-lowering in difficult-to-treat patients with hypercholesterolemia. Am J Ther. 2005;12(4):306-310.

47. McKenney J, Jones M, Abby S. Safety and efficacy of colesevelam hydrochloride in combination with fenofibrate for the treatment ofmixed hyperlipidemia. Curr Med Res Opin. 2005;21:1403-1412.

48. Garg A, Grundy SM. Cholestyramine therapy for dyslipidemia in non-insulin-dependent diabetes mellitus. A short-term, double-blind, crossover trial. Ann Intern Med. 1994;121:416-422.
49. Zieve FJ, Kalin MF, Schwartz SL, et al. Results of the glucose-lowering effect of WelChol study GLOWS: a randomized, double-blind, placebo-controlled pilot study evaluating the effect of colesevelam hydrochloride on glycemic control in subjects with type 2 diabetes. Clin Ther. 2007;29:74-83.

50. Fonseca VA, Rosenstock J, Wang AC, Truitt KE, Jones MR. Colesevelam $\mathrm{HCl}$ improves glycemic control and reduces LDL cholesterol in patients with inadequately controlled type 2 diabetes on sulfonylurea-based therapy. Diabetes Care. 2008;31(8):1479-1484.

51. Bays HE, Goldberg RB, Truitt KE, Jones MR. Colesevelam hydrochloride therapy in patients with type 2 diabetes mellitus treated with metformin: glucose and lipid effects. Arch Intern Med. 2008;168(18):1975-1983.

52. Devaraj S, Autret B, Jialal I. Effects of colesevelam hydrochloride (WelChol) on biomarkers of inflammation in patients with mild hypercholesterolemia. Am J Cardiol. 2006;98(5):641-643.

53. Donovan JM, Stypinski D, Stiles MR, Olson TA, Burke SK. Drug interactions with colesevelam hydrochloride, a novel, potent lipid-lowering agent. Cardiovasc Drugs Ther. 2000;14(6):681-690.

54. Welchol prescribing information. Available at http://www.welchol. com/pdf/Welchol_PI.pdf.

55. Thomson AB, Keelan M. Feeding rats diets containing cheno1365-71 or ursodeoxycholic acid or cholestyramine modifies intestinal uptake of glucose and lipids. Digestion. 1987;38(3):160-170.

56. Brand SJ, Morgan RG. Stimulation of pancreatic secretion and growth in the rat after feeding cholestyramine. Gastroenterology. 1982;83:851-859.

57. Staels B, Kuipers F. Bile acid sequestrants and the treatment of type 2 diabetes mellitus. Drugs. 2007;67(10):1383-1392.

58. Nathan DM, Davidson MB, DeFronzo RA, et al. American Diabetes Association. Impaired fasting glucose and impaired glucose tolerance: implications for care. Diabetes Care. 2007;30(3):753-759. 
